INPLASY

PROTOCOL

To cite: Singh et al. Efficacy and safety of Ayurveda interventions for Epilepsy : A systematic review. Inplasy protocol 202130082. doi: 10.37766/inplasy2021.3.0082

Received: 23 March 2021

Published: 23 March 2021

Corresponding author:

Shyam Babu Singh

dr.shyam84@yahoo.in

Author Affiliation:

Regional Ayurveda Research Institue, Aamkho, Gwalior, (M.P), India.

Support: CCRAS, New Delhi, India.

Review Stage at time of this submission: Preliminary searches.

Conflicts of interest: None declared.

\section{Efficacy and safety of Ayurveda interventions for Epilepsy : A systematic review}

Singh, SB1; Ahmad, A2; Prakash, O3; Kumar, V4.

Review question / Objective: This review seeks to establish, through the available literature, the effectiveness and safety of Ayurveda intervention in the management of Epilepsy (mentioned as Apasmara in Ayurveda) The specific review question to be addressed are: "Are Ayurvedic interventions are effective and safe as standalone or adjunctive therapy in the management of Epilepsy (Apasmara) in comparison to the standard of care treatment?".

Main outcome(s): Primary Response to treatment (improvement in Subjective and/or objective criteria of assessment) Secondary 1. Serious adverse events (resulting in death, disability or incapacity, complications, were lifethreatening, led to hospitalization or prolong of hospitalization) 2. Reported improvement in the patients health-related quality of life.

INPLASY registration number: This protocol was registered with the International Platform of Registered Systematic Review and Meta-Analysis Protocols (INPLASY) on 23 March 2021 and was last updated on 23 March 2021 (registration number INPLASY202130082).

\section{INTRODUCTION}

Review question / Objective: This review seeks to establish, through the available literature, the effectiveness and safety of Ayurveda intervention in the management of Epilepsy (mentioned as Apasmara in Ayurveda) The specific review question to be addressed are: "Are Ayurvedic interventions are effective and safe as standalone or adjunctive therapy in the management of Epilepsy (Apasmara) in 
comparison to the standard of care treatment?".

Rationale: Epilepsy is one of the world's oldest recognized conditions, with written records dating back to $4000 \mathrm{BC}$. Fear, misunderstanding, discrimination and social stigma have surrounded epilepsy for centuries. This stigma continues in many countries today and can impact the quality of life for people with the disease and their families. It is a chronic non-communicable disease of the brain that affects more than 70 million people worldwide. It is characterized by recurrent seizures, which are brief episodes of involuntary movement that may involve a part of the body (partial) or the entire body (generalized) and are sometimes accompanied by loss of consciousness and control of bowel or bladder function. In Ayurveda it is mentioned as Apasmara and its management is also described there. There are plenty of studies showing the promising results of Shodhan and Shaman treatment in the management of Epilepsy (Apasmar) but no systematic review over the subject has been published yet. So the present work aims towards the systematic review of all published data of Ayurveda intervention in the management of Epilepsy. This will help to assess the safety and effectiveness of Ayurveda interventions for the management of epilepsy. This systematic review would probably be helpful to ensure the role and significance of Ayurveda interventions in the management of Epilepsy.

Condition being studied: Epilepsy: Modern medicine perspective Epilepsy is a chronic neurological disease that, although occurring intermittently, can have lasting changes in the neural circuitry. The disease has a strong propensity for comorbidities including psychiatric and cognitive impairments. International League Against Epilepsy (ILAE) defines epilepsy as "a disorder of the brain characterized by an enduring predisposition to generate epileptic seizures and by the neurobiologic, cognitive, psychological and social consequences" and an epileptic seizure as "a transient occurrence of signs and/or symptoms due to abnormal excessive or synchronous neuronal activity in the brain. The cause of most cases of epilepsy is unknown. Some cases occur as the result of brain injury, stroke, brain tumors, infections of the brain, and birth defects through a process known as epileptogenesis. Known genetic mutations are directly linked to a small proportion of cases. Epileptic seizures are the result of excessive and abnormal neuronal activity in the cortex of the brain. Epileptic seizures are characterized as focal and general seizures. Focal seizure activity is limited to a small part of the brain hemisphere and these are of two types i.e seizures with retained awareness and seizures with loss of awareness. General seizures occur when there is widespread seizure activity in the left and right hemispheres of the brain. General seizures are classified as petit mal, Grand mal, tonic seizures, clonic seizures, etc. Epilepsy: Ayurveda Perspective In most Ayurvedic classical texts, epilepsy has been mentioned as Apasmara or Apasmrti, and it has been mentioned as one of the earliest eight diseases known (diagnosed) that can be controlled only with medical therapies and can sometimes be incurable and remain uncontrolled. Ayurveda defines epilepsy as a group of symptoms like temporary loss of consciousness, darkness, fits, and ghastly gesticulation. Ayurveda believes that due to prolonged use of unwholesome and unhygienic dietary habits, psychological factors like excessive grief, hunger, anger, worry, passion, anxiety, and excitement, Tama and Rajo Dosha are aggravated and Satva guna becomes weak, individual becomes psychologically weak and unhealthy and Apasmara is caused. Prodromal symptoms of the disease are contraction of eyebrows, constant irregular movement of eyes, and hearing of such sounds as are non-existent, excessive discharge of saliva and nasal excreta, disinclination for food (anorexia and indigestion), distension of abdomen, body aches, transient blackout, giddiness, profuse sweating, increased thirst, fainting, hallucinations, falling and insomnia. Clinical manifestations of epilepsy (Rupa) are categorized into four types according to 
the dominant Dosa (humour) involved in its pathogenesis: Vataja, Pittaja, Kaphaja, and Sannipataja. The Vataja type is characterized by frequent fits, regaining consciousness in the shortest time interval; bulging eyes; excessive crying, frothing at the mouth; irregularly contracted fingers; reddish rough and blackish nails, eyes, face, and skin; hallucinations and trembling. Kaphaja type has features of prolonged fits with delayed recovery; increased frothing at the mouth; white nails, eyes, face, and skin; and visions of white, heavy, unctuous, smooth objects (description like grand mal seizures). Pittaja type is characterized by regaining consciousness in shorter periods, scratching of ground, greenish-yellow and coppery nails, eyes, face, and skin; and visions of bloody, agitated, irritated, frightful, and burning objects (description like partial complex seizures). The fourth type, the Sannipatika form of the disease, is caused by the simultaneous vitiation of all the three doshas that gives rise to a combination of symptoms and signs and is incurable (description like intractable epilepsy).

\section{METHODS}

Search strategy: Search strategy combining MeSH terms and free text words using the Boolean operators "AND" and "OR" will be used such as (Ayurveda OR Ayurvedic therapy OR Ayurvedic treatment OR Herbal Ayurveda OR Polyherbal OR Panchakarma OR Rasaushadhi OR Rasakalpa OR Herbo-mineral OR Metallic Ayurveda OR Mercurial OR Plants OR Herbs OR Madhu OR Traditional Medicine OR Alternative Medicine OR Complementary Medicine Ayurveda OR Massage OR Shirodhara OR Leech OR Vaman OR Virechan OR Basti OR Siravedh OR Lepa OR Tel OR Oil OR Jalauka OR Vati OR Guti OR Kwath OR AND (Epilepsy OR Apasmara AND (Clinical trial) as title, abstract or keyword. There were not any restrictions in language, publication date, or type.

Participant or population: Published Clinical trials studying efficacy \&/or safety of Ayurvedic Interventions in Epilepsy. (may be diagnosed Apasmara, mentioned in Ayurveda).

Intervention: Ayurvedic interventions including Shodhan (biopurification procedures) or Shodhan and Shamana (internal and external medication) with any dose, type, schedule, drug, dosage form as standalone therapy.

Comparator: 1. Ayurveda Treatment (Shamana or/and Shodhana) with different dose, type, schedule, medicine, medicine form as compare to intervention(s)/ exposure(s). 2. No treatment or Placebo and/or Sham therapy 3. Non-Ayurveda interventions or combination of Ayurveda and non Ayurveda interventions.

Study designs to be included: • Randomized Controlled Trial (RCT) - Nonrandomized Controlled Trial (NRCT) - Quasi Randomized Controlled Trial (QRCT) • Controlled Clinical Trial (CCT) • Randomized/Nonrandomised Placebo control trials - Pragmatic trials - Controlled Before and After Study (CBA) - Parallel arm clinical trials.

Eligibility criteria: We will include studies that have participants diagnosed as cases of Epilepsy of any type, of any age of both sexes.

Information sources: We will search the following databases: Electronic database Complementary and alternative medicine (CAM) databases HerbMed - Cochrane Complementary Medicine trial register • Annotated Bibliography of Indian Medicine - Open access databases - Scholarly exchange - Free Medical Journals database - Official publications (journals) of various Indian societies / associations - Open Access and Institutional Repositories, with E Prints ((http://www.eprints.org/) - Main stream Allopathic databases - PubMed and CAM on Pub Med - Cochrane library http:// www.cochranelibrary.com/ - Indian databases - National Institute of Science Communication and Information Resources (NISCAIR) and the NISCAIR project register - IndMED - DHARA (Digital Helpline for 
Ayurveda Research Abstracts http:// www.dharaonline.org/Forms/Home.aspx) • AYUSH Research Portal (http:// ayushportal.nic.in/) which is maintained by the Council Council for Research in Ayurvedic Sciences, Ministry of AYUSH, Government of India - South Asian Database of Controlled Clinical Trials (www.cochrane-sadcct.org) - For ongoing trials- clinical trial registry of Indiactri.nic.in - World Health Organizations International Clinical Trials Registry Platform -http://apps.who.int/trialsearch/ • For dissertations in the public domain: http://shodhganga.inflibnet.ac.in/simplesearch Handsearching Hand searching for journals that may not have been indexed in any electronic database or may have been indexed in such a way that database searching is impractical. - Baghels thesis list- researches in Ayurveda - Ayurvedic Research Database (ARD) https:// www.ayurvedahealthcare.info/content/ ayurveda-research-database-ard Ayurvedic journals published from India https://www.ayurvedahealthcare.info/ ayurveda-journals - Conference proceedings/ reports/ compendia.

Main outcome(s): Primary Response to treatment (improvement in Subjective and/ or objective criteria of assessment) Secondary 1. Serious adverse events (resulting in death, disability or incapacity, complications, were life-threatening, led to hospitalization or prolong of hospitalization) 2. Reported improvement in the patients health-related quality of life.

Additional outcome(s): Withdrawals due to adverse events or lack of efficacy or inconvenience of therapy/treatment.

Data management: We will extract and record data on study characteristics including methods, participants, interventions, and outcomes using the PICOT table. The authors of the trials with insufficient or missing data will be contacted to get more information. We will resolve any disagreements by referring to the trial report and through discussion.
Quality assessment / Risk of bias analysis: Two reviewers will independently assess the risk of bias in included studies. Randomized controlled trials will be assessed with the help of the Cochrane tool of Risk of bias and that of nonrandomized trials will be assessed with ROBINS-I tool (Risk Of Bias In Nonrandomized Studies-of Interventions). Disagreements between these reviewers will be resolved by discussion, with the involvement of a third reviewer if necessary and the results will be interpreted. Two reviewers will assess the quality of reporting trial independently. Assessment will be performed under three categories, "Yes" reporting, "No" reporting, and "Incomplete" reporting. Two points will be given for each item if it is reported completely, in case of incomplete reporting only one point will be given to that item and no point for "No" reporting. Results will be interpreted in terms of percentage (\%) of the mean of each three category reporting items. If any disagreement still persists causing difficulty in data extraction clarification will be sought from the authors of the trial.

Strategy of data synthesis: Two investigators including a consultant (statistics) will analyze data by using appropriate software. Dichotomous data will be presented and combined using relative risks, continuous data will be summarized by arithmetic means, and standard deviations data will be combined by using weighted mean differences; both will be accompanied by $95 \%$ confidence intervals. Medians and ranges will be reported in tables. Arithmetic means and standard deviations will be used to summarize continuous data when the data are assumed to be normally distributed. Separate summary effect estimates will also be generated for studies that meet and do not meet the individual quality criterion. Heterogeneity among trials will be assessed by inspecting forest plots, to look for overlapping confidence intervals, applying the chi-squared test, with a Pvalue of 0.05 , indicating statistical significance, and using the I 2 test with a value of $50 \%$, used to denote moderate 
levels of heterogeneity. If heterogeneity is detected and it is still considered clinically meaningful to combine studies, then a random-effects model will be used. A sensitivity analysis, to investigate the robustness of the results to the quality components will be done provided there are sufficient trials. A funnel plot will be utilized to indicate publication bias, heterogeneity of results, or differences in the methodological quality.

Subgroup analysis: On the basis of retrieved data subgroup analysis will be done according to the severity of disease (epilepsy), treatment provided(procedural interventions, non-procedural interventions, or both), types of control(no treatment/placebo/non - Ayurveda interventions or combinations), duration of the treatment provided, age groups, etc.

Sensitivity analysis: If there are sufficient studies, a sensitivity analysis will be performed by the 'leave-one-out' method to check the effect of individual study on over results of the analysis.

Language: Data available in English, Hindi or Indian Regional Languages will be included in this review because of unavailability of other language translation.

Country(ies) involved: India.

Keywords: Ayurveda, Epilepsy, Apasmara, Systematic review, protocol.

Contributions of each author:

Author 1 - Shyam Babu Singh - Protocol designing and drafted the manuscript.

Email: dr.shyma84@yahoo.in

Author 2 - Azeem Ahmad - Conceived the concept, Protocol designing, manuscript reviewing.

Email: azeem2905@gmail.com

Author 3 - Om Prakash - Guidance and support.

Email: oprakash749@gmail.com

Author 4 - Vijay Kumar - Protocol designing and drafted the manuscript.

Email: vijaychem99@gmail.com 